\title{
Thymosin beta 4 ophthalmic solution for dry eye: a randomized, placebo-controlled, Phase II clinical trial conducted using the controlled adverse environment $\left(\mathrm{CAE}^{\mathrm{TM}}\right)$ model
}

\author{
This article was published in the following Dove Press journal: \\ Clinical Ophthalmology \\ 20 May 2015 \\ Number of times this article has been viewed
}

\section{Gabriel Sosne' \\ George W Ousler}

'Kresge Eye Institute, Wayne State University, Detroit, MI, ${ }^{2}$ Ora Inc, Andover, MA, USA
Correspondence: Gabriel Sosne Departments of Ophthalmology and Anatomy and Cell Biology, Kresge Eye Institute, Wayne State University School of Medicine, 4717 St Antoine Detroit, MI 4820I, USA

Tel + I 3 | 3577 I356

Fax + I 3135773125

Email gsosne@med.wayne.edu
Background: The purpose of this study was to evaluate the safety and efficacy of thymosin beta 4 ophthalmic solution (RGN-259; T $\beta 4$ ) in subjects with moderate to severe dry eye using the CAETM model.

Methods: This single-center, prospective, double-masked, placebo-controlled Phase II study randomized 72 qualifying subjects $1: 1$ to receive either $0.1 \% \mathrm{~T} \beta 4$ or placebo treatment for a total of 28 days. The study consisted of six visits over a 32-day period, including a screening visit (day -1), controlled adverse environment challenge (CAE) visits (day 1, day 28), and follow-up visits (days 14, 29, and 30). The primary efficacy endpoints were ocular discomfort scores and inferior corneal staining measured at visit 5 on day 29. Secondary endpoints included central and superior corneal staining, conjunctival staining, conjunctival redness, tear-film break-up time, and daily symptom scores recorded over the course of the study. Safety measures included visual acuity, slit-lamp evaluation, conjunctival redness, tear film break-up time, intraocular pressure, dilated funduscopy, and corneal sensitivity.

Results: Neither of the primary endpoints, ie, ocular discomfort or inferior corneal staining, showed a significant difference between treatment and control groups at visit 5. Despite this, significant differences between treatment groups were observed for a number of secondary endpoints. The discomfort scores in the CAE on day 28 were reduced by $27 \%$ in $0.1 \% \mathrm{~T} \beta 4$-treated subjects compared with the placebo group $(P=0.0244)$. Subjects in the $0.1 \% \mathrm{~T} \beta 4$ treatment group also showed statistically significant improvements in central and superior corneal staining compared with staining scores in the control group $(P=0.0075$ and $P=0.0210)$. No adverse events were observed.

Conclusion: This study confirms the efficacy of $0.1 \% \mathrm{~T} \beta 4$ as a topical treatment for relief of signs and symptoms of dry eye. Significant improvements in both signs and symptoms of dry eye were observed, and the treatment exhibited a large safety window, with no adverse events reported by any subjects enrolled in the study.

Keywords: thymosin beta 4, RGN-259, controlled adverse environment, dry eye

\section{Introduction}

Dry eye is a common ocular disorder that can range from a minor discomfort to a significant disease; in the most severe cases, it can lead to corneal ulcers, infections, and serious visual impairment. ${ }^{1}$ Conventional treatments using artificial tears, ointments, serum tears, or anti-inflammatories provide limited relief, and there is a real need for improved treatments. A therapeutic agent that could reduce inflammation and accelerate ocular surface healing would reduce the risk of permanent injury, improve vision, and provide improved comfort for those with dry eye. 
Thymosin beta 4 (TR4) is a 43-amino acid peptide that is a major constituent protein of platelets, macrophages, and polymorphonuclear cells, where it acts as a G-actin binding molecule and regulator of actin polymerization..$^{2-5}$ These cell types function in trauma response and wound repair, and $\mathrm{T} \beta 4$ is among the first genes to be upregulated in the integrated physiological response to tissue trauma. ${ }^{2,4}$ Endogenous or exogenous $\mathrm{T} \beta 4$ promotes wound repair in dermal, ocular, cardiac, and central nervous system animal models, ${ }^{6-13}$ and accelerates dermal repair in several animal models. ${ }^{7,14} \mathrm{~T} \beta 4$ is a pleotropic signaling molecule that works in part by downregulating nuclear factor kappa B-mediated transcription of inflammatory chemokines, cytokines, and metalloproteinases. ${ }^{2,4,15}$ It also acts to upregulate expression of laminin-5, and promotes cell migration, cell survival, and recruitment and maturation of stem cells..$^{6-8,12,14-16}$ This spectrum of activity makes $\mathrm{T} \beta 4$ an attractive molecule as a potential therapeutic agent for inflammatory or traumatic conditions.

The effect of T $\beta 4$ on corneal ocular surface healing has been examined in both rats and mice following corneal injury. ${ }^{8,10,11,18} \mathrm{~T} \beta 4$ promoted corneal ocular surface healing, increased corneal epithelial cell migration, and decreased proinflammatory cytokine levels in multiple rodent models of corneal injury..$^{10,11,18}$ Mouse corneas topically treated with $\mathrm{T} \beta 4$ after alkali injury demonstrated accelerated re-epithelialization at all time points and decreased polymorphonuclear infiltration at 7 days post-injury compared with vehicle-treated controls. ${ }^{11}$ Other murine model studies demonstrated that $\mathrm{T} \beta 4$ promoted improved corneal epithelial intercellular adhesion following corneal dry eye injury. ${ }^{20}$ The results of these studies show that $\mathrm{T} \beta 4$ reduced corneal staining more than positive controls and demonstrated a statistically significant reduction in staining compared with vehicle control.

The clinical potential of topical $0.1 \% \mathrm{~T} \beta 4$ for treatment of corneal inflammatory conditions was demonstrated in a recent study testing treatment of neurotrophic keratitis, a condition marked by persistent, non-healing corneal defects that are typically unresponsive to available anti-inflammatory agents. ${ }^{21}$ This was a physician-sponsored compassionate use trial using the RGN-259 formulation of $0.1 \% \mathrm{~T} \beta 4$, and a complete clearing of defects was observed in six of nine subjects. Another physician-sponsored study examined the efficacy of $0.1 \% \mathrm{~T} \beta 4$ in subjects with severe dry including several with graft versus host disease. ${ }^{22}$ Subjects in this study showed significant improvements in both ocular discomfort and total corneal fluorescein staining. ${ }^{22}$ These studies established the potential of topical T $\beta 4$ as a therapeutic approach to ocular surface disorders. The safety of topical $\mathrm{T} \beta 4$ formulations has been demonstrated, both in dermal preparations and in the preservative-free formulation that has been used in the eye. In a Phase I clinical trial, an injectable solution of T $\beta 4$ administered for 14 consecutive days at four escalating dose levels was deemed safe and well tolerated. ${ }^{23}$

The established anti-inflammatory and tissue repair actions of $\mathrm{T} \beta 4$, together with evidence from preclinical and clinical studies, suggest that $\mathrm{T} \beta 4$ may represent an important new therapy for treatment of dry eye. Here, we describe a Phase II study in subjects with moderate to severe dry eye, exacerbated by a controlled adverse environment (CAETM). The use of the CAE allows for a greater standardization of environmental conditions that are a key component of dry eye, while at the same time providing a greater therapeutic window to assess therapy efficacy. ${ }^{24}$

\section{Materials and methods}

This study employed a single-center, randomized, doublemasked, placebo-controlled protocol with equal randomization of subjects into two treatment groups, ie, $0.1 \% \mathrm{~T} \beta 4$ and placebo, in 72 subjects. The demographics of the subjects are shown in Table 1. The study was conducted in compliance with good clinical practices, including International Conference on Harmonisation guidelines, and consistent with the 1989 version of the Declaration of Helsinki. The institutional review board, Alpha IRB, San Clemente, CA, USA, approved the protocol.

Solid phase chemical synthesis was used to prepare the $\mathrm{T} \beta 4$; this was followed by reversed-phase high performance liquid chromatographic purification and lyophilization of the acetate salt. The test agents were formulated as preservativefree, sterile solutions, with the active containing $0.1 \%(\mathrm{w} / \mathrm{w})$ $\mathrm{T} \beta 4$. Both active and placebo were packaged in $8 \mathrm{~mL}$ squeeze bottles that contained approximately $2 \mathrm{~mL}$ solution. The placebo formulation was composed of the same excipients as the active, and was identical to the active in color, consistency, and odor. Each bottle of test agent delivered the same amount per drop, and had to be used within 6 hours of opening; therefore, two bottles were needed for each day of dosing.

\section{Study design}

Enrolled subjects were aged 18 years or older, of any race and sex, with a history of dry eye syndrome in both eyes, and use or desire to use artificial tear substitutes within the previous 6 months. All had corneal staining of $\geq 2$ in any corneal surface segment (at least one eye), and a conjunctival redness 
Table I Patient demographic characteristics

\begin{tabular}{|c|c|c|c|}
\hline Characteristic & $\begin{array}{l}0.1 \% \text { T } \beta 4 \\
(n=36)\end{array}$ & $\begin{array}{l}\text { Placebo } \\
(n=36)\end{array}$ & $\begin{array}{l}\text { Total ITT, safety } \\
\text { population }(n=72)\end{array}$ \\
\hline Mean age, years (SD) & $57.1(12.07)$ & $55.3(12.76)$ & $56.2(12.37)$ \\
\hline Female, $\mathrm{n}^{\mathrm{a}}(\%)$ & $29(80.6 \%)$ & $25(69.4 \%)$ & $54(75.0 \%)$ \\
\hline Male, $\mathrm{n}^{\mathrm{a}}(\%)$ & 7 (19.4\%) & II (30.6\%) & $18(25.0 \%)$ \\
\hline White, $\mathrm{n}^{\mathrm{a}}(\%)$ & $33(91.7 \%)$ & 34 (94.4\%) & 67 (93.1\%) \\
\hline Asian, $\mathrm{n}^{\mathrm{a}}(\%)$ & $2(5.6 \%)$ & $\mathrm{I}(2.8 \%)$ & $3(4.2 \%)$ \\
\hline Black or African American, $\mathrm{n}^{\mathrm{a}}(\%)$ & $0(0.0 \%)$ & I (2.8\%) & $\mathrm{I}(\mathrm{I} .4 \%)$ \\
\hline Other, $\mathrm{n}^{\mathrm{a}}(\%)$ & $\mathrm{I}(2.8 \%)$ & $0(0.0 \%)$ & $\mathrm{I}(\mathrm{l} .4 \%)$ \\
\hline Non-Hispanic, $\mathrm{n}^{\mathrm{a}}(\%)$ & $34(94.4 \%)$ & $36(100.0 \%)$ & 70 (97.2\%) \\
\hline \multicolumn{4}{|l|}{ Iris color, $\mathrm{n}^{\mathrm{b}}(\%)$} \\
\hline Black & $4(5.6 \%)$ & $0(0.0 \%)$ & $4(2.8 \%)$ \\
\hline Blue & $28(38.9 \%)$ & $30(41.7 \%)$ & $58(40.3 \%)$ \\
\hline Brown & $28(38.9 \%)$ & $26(36.1 \%)$ & $54(37.5 \%)$ \\
\hline Hazel & $6(8.3 \%)$ & $8(11.1 \%)$ & $14(9.7 \%)$ \\
\hline Green & $6(8.3 \%)$ & $8(11.1 \%)$ & 14 (9.7\%) \\
\hline Other & $0(0.0 \%)$ & $0(0.0 \%)$ & $0(0.0 \%)$ \\
\hline
\end{tabular}

Notes: $n^{\mathrm{a}}$, number of subjects; $\mathrm{n}^{\mathrm{b}}$, number of eyes.

Abbreviation: ITT, intention to treat.

score of $\geq 1$ (at least one eye) prior to CAE at visit 1 . Subjects also had a best corrected visual acuity of $0.7 \log$ MAR or better in each eye, and had to demonstrate a positive response to the $\mathrm{CAE}$ as defined in the protocol at visit 1 for inclusion. All participants provided informed consent. The study comprised six visits conducted over a period of approximately 31 days (Figure 1 and Table 1).

Scales for corneal staining were based upon a $0-4$ rating where $0=$ no staining and $4=$ confluent staining. Conjunctival redness was measured using a similar $0-4$ scale, where $0=$ none and $4=$ severe. In both cases, 0.5 incremental scores were allowed. Ocular discomfort was graded on a $0-4$ scale with no half units; for this scale $0=$ no discomfort and $4=$ constant discomfort.

At visit 1 (day -1 ) prospective subjects had their signs and symptoms assessed before and after CAE exposure. Ocular discomfort scores were recorded throughout the CAE challenge. Subjects initially qualifying for study entry were instructed to discontinue all ophthalmic medications and instilled a commercially available sterile irrigating (balanced salt) solution (ie, the run-in solution) twice daily until visit 2 , day 0 . The first dose of this run-in solution was administered in-office by a trained technician. Subjects were dispensed a daily diary with which to enter the details of dosing and symptomatology. At visit 2, the 24-hour follow-up visit, subjects were reassessed and those who met entry criteria for discomfort and corneal staining were randomized to receive either $0.1 \% \mathrm{~T} \beta 4$ ophthalmic solution (RGN-259; RegeneRx Pharmaceuticals Inc, Rockville, MD, USA) or placebo, for the 28-day duration of test agent treatment. Subjects received their first dose of randomized test agent in-office at visit 2 , and were then instructed to instill 1-2 drops of study drug $(0.1 \% \mathrm{~T} \beta 4$ or placebo $)$ into each eye twice a day for a total of 28 days.

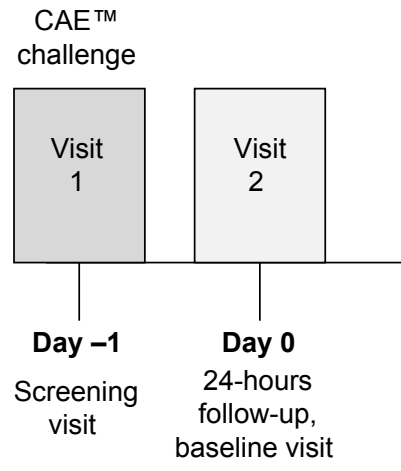

Figure I Study timeline.

Abbreviation: CAE, controlled adverse environment. 
Subjects returned to the clinic for visit 3 (day 14 \pm 2 ) to have their signs and symptoms assessed, and to receive additional supplies of test agent. At visit 4 (day 28 2 ) subjects underwent a second CAE exposure; on the following two days, visits 5 and 6 , subjects underwent additional assessments of signs and symptoms.

The coprimary endpoints for this study were the differences at visit 5 between the active and placebo mean values for inferior corneal staining (primary sign) and for ocular discomfort score (primary symptom). Secondary endpoints included differences at visit 5 in corneal and conjunctival staining, conjunctival redness, and tear film break-up time; differences at visit 4 in ocular discomfort during CAE, corneal and conjunctival staining, conjunctival redness, and tear film break-up time.

\section{Statistical analysis}

The quantitative variables were summarized using summary statistics (n, mean, median, standard deviation, minimum, and maximum). Qualitative variables were summarized using counts and percentages. Inferior corneal fluorescein staining score at visit 5 (primary sign) and ocular discomfort at visit 5 (primary symptom) were summarized using descriptive statistics (number of observations, mean, standard deviation, median, minimum, and maximum). Active treatment was compared with placebo using a two-sample $t$-test assuming unequal variances, assessed at the level of $\alpha=0.05$. The primary efficacy variables were also analyzed using a nonparametric Wilcoxon rank sum test for sensitivity, as well as an analysis of covariance (ANCOVA) model adjusted for baseline (visit 1 pre-CAE) score. The change from baseline to visit 5 was also analyzed.

The continuous secondary efficacy variables collected at each visit were also summarized statistically (n, mean, standard deviation, median, minimum, and maximum), and analyzed similarly to the primary efficacy endpoints. All visit-based data were analyzed at each time point (pre- and post-CAE) if applicable, as well as the change from pre-CAE to post-CAE. Finally, a repeated-measures mixed model was generated to assess the environmental treatment effect, including data from visit 3 , visit 4 (pre-CAE), visit 5, and visit 6 . Baseline (visit 1 pre-CAE) was included as a covariate, and the model was used to obtain least squares estimates and test for treatment effects at each visit and overall. Ocular discomfort in the chamber was measured every 5 minutes after the patient entered the CAE. The area under the curve was calculated for each patient using the trapezoidal rule, and compared between treatment groups using a two-sample $t$-test assuming unequal variances, as well as an ANCOVA model adjusted for baseline (visit 1 area under the curve). Each symptom collected in the diary was summarized by day and time point, and compared between groups using a mixed model accounting for repeated measures within each subject.

\section{Safety analysis}

The quantitative safety variables were summarized using descriptive statistics (n, mean, standard deviation, minimum, median, and maximum). The qualitative safety variables were summarized by frequencies and percentages. Adverse events were coded using the MedDRA dictionary, version 13.1. Frequencies and percentages were provided by treatment group of subjects with treatment-emergent adverse events (TEAEs), serious TEAEs, and TEAEs causing premature discontinuation. An adverse event was treatment-emergent if it occurred or worsened after the first dose of study drug up through visit 6 .

Safety endpoints were analyzed for both eyes. For efficacy endpoints, the unit of analysis was the "worst eye". Eyes were eligible for analysis if they met all "inclusion criteria". In the event that both eyes were eligible for analysis, the worst eye was chosen as the eye with the greater increase of inferior corneal staining from pre-CAE at visit 1 to visit 2 . If both eyes had an equal increase in inferior corneal staining from pre-CAE at visit 1 to visit 2, the eye with greater ocular discomfort at visit 2 was chosen as the worst eye. If both eyes had an equal increase in inferior corneal staining from preCAE at visit 1 to visit 2 and equal ocular discomfort at visit 2 , then the right eye was chosen as the worst eye.

\section{Results}

A total of 72 subjects were screened. Of these, 72 were enrolled in the study, with 36 subjects assigned to each group, ie, $0.1 \% \mathrm{~T} \beta 4$ ophthalmic solution or placebo. Sixty-nine subjects completed the trial. Two subjects in the placebo group and one in the $0.1 \% \mathrm{~T} \beta 4$ did not complete the study and five subjects had major protocol deviations and were not included in the per-protocol analysis. Subject demographics are summarized in Table 2. All subjects received the study medication or vehicle on day 0 (visit 2).

The primary efficacy measures were inferior fluorescein corneal staining and ocular discomfort in the worst eye at visit 5. Mean inferior fluorescein corneal staining was 2.08 in $0.1 \% \mathrm{~T} \beta 4$-treated subjects and 1.90 in placebo-treated subjects. This difference (0.18) was not statistically significant $(P=0.2586$, Wilcoxon rank sum test). The ANCOVA with 
Table 2 Schedule of procedures

\begin{tabular}{|c|c|c|c|c|c|c|c|c|c|c|}
\hline \multirow[t]{3}{*}{ Procedure } & \multicolumn{3}{|c|}{ Visit I (day-I) } & \multirow{3}{*}{$\begin{array}{l}\begin{array}{l}\text { Visit } 2 \\
(\text { day } 0)\end{array} \\
\begin{array}{l}24-h o u r \\
\text { follow-up }\end{array}\end{array}$} & \multirow{3}{*}{$\begin{array}{l}\begin{array}{l}\text { Visit } 3 \\
\text { (day I4 } \pm 2 \text { ) }\end{array} \\
\text { Day I4 } \\
\text { follow-up }\end{array}$} & \multicolumn{3}{|c|}{ Visit 4 (day $28 \pm 2$ ) } & \multirow{3}{*}{$\begin{array}{l}\text { Visit } 5 \\
\text { (day 29) } \\
\text { 24-hour } \\
\text { follow-up }\end{array}$} & \multirow{3}{*}{$\begin{array}{l}\text { Visit } 6 \\
\text { (day } 30 \text { ) } \\
48 \text {-hour } \\
\text { follow-up }\end{array}$} \\
\hline & \multicolumn{3}{|c|}{ CAE challenge } & & & \multicolumn{3}{|c|}{ CAE challenge } & & \\
\hline & Pre-CAE & CAE & Post-CAE & & & Pre-CAE & CAE & Post-CAE & & \\
\hline Informed consent & $\times$ & & & & & & & & & \\
\hline Medical history & $x$ & & & $\times$ & $\times$ & $\times$ & & & $x$ & $\times$ \\
\hline Pregnancy test & $x$ & & & & & & & & & $\times$ \\
\hline Randomization & & & & $\times$ & & & & & & \\
\hline Dispense study drug & & & & $\times$ & $\times$ & & & $\times$ & $\times$ & \\
\hline Dispense I-day diary & & & $\times$ & & & & & $\times$ & $\times$ & \\
\hline Collect I-day diary & & & & $\times$ & & & & & $x$ & $\times$ \\
\hline Dispense 2-week diary & & & & $\times$ & $\times$ & & & & & \\
\hline Collect 2-week diary & & & & & $\times$ & $\times$ & & & & \\
\hline 4-symptom evaluation & $\times$ & & & $\times$ & $\times$ & $x$ & & & $\times$ & $\times$ \\
\hline VA & $\times$ & & & $\times$ & $\times$ & $\times$ & & & $\times$ & $\times$ \\
\hline Slit-lamp examination & $\times$ & & $\times$ & $\times$ & $\times$ & $\times$ & & $\times$ & $\times$ & $\times$ \\
\hline Conjunctival redness & $\times$ & & $\times$ & $\times$ & $\times$ & $\times$ & & $\times$ & $x$ & $x$ \\
\hline TBUT & $\times$ & & $\times$ & $\times$ & $\times$ & $x$ & & $\times$ & $\times$ & $\times$ \\
\hline Corneal staining & $\times$ & & $\times$ & $\times$ & $\times$ & $\times$ & & $\times$ & $\times$ & $\times$ \\
\hline Conjunctival staining & $\times$ & & $\times$ & $\times$ & $x$ & $x$ & & $\times$ & $x$ & $\times$ \\
\hline Ocular discomfort & $\times$ & $\times$ & & $\times$ & $\times$ & $\times$ & $x$ & & $\times$ & $\times$ \\
\hline IOP, dilated funduscopy & & & & $\times$ & & & & & & $\times$ \\
\hline Corneal sensitivity & $\times$ & & & & & $\times$ & & & & \\
\hline Adverse event query & & & $\times$ & $\times$ & $\times$ & $x$ & & $\times$ & $\times$ & $\times$ \\
\hline Study exit & & & & & & & & & & $x$ \\
\hline
\end{tabular}

Abbreviations: CAE, controlled adverse environment; VA, visual acuity; TBUT, tear break-up time; IOP, intraocular pressure.

visit 2 values as the covariate provided least square means of 2.06 in $0.1 \%$ T $\beta 4$-treated subjects and 1.92 in placebo-treated subjects. This difference $(0.14)$ was also not significant $(P=0.3452)$. Mean ocular discomfort at visit 5 was 1.6 in $0.1 \% \mathrm{~T} \beta 4$-treated eyes and 1.3 in placebo-treated eyes. This difference $(0.3)$ was not statistically significant $(P=0.2210)$. The ANCOVA with visit 2 as the covariate provided a least square mean of 1.5 , again with a difference $(0.2)$ that was not statistically significant $(P=0.4901)$. Despite these findings, when measures were adjusted for baseline in several ad hoc statistical analyses, several significant differences between active and placebo groups were identified.

To address the possible impact of baseline staining, we compared superior corneal staining scores from the 2 days of CAE exposure (visits 1 and 4), before or after 28 days of treatment with $0.1 \% \mathrm{~T} \beta 4$ or placebo; the results of this analysis are shown in Figure 2. Mean superior corneal staining after CAE was reduced after 28 days of $0.1 \% \mathrm{~T} \beta 4$ treatment, while those in the placebo group showed a modest (insignificant) increase in mean staining. Comparison of the change in staining demonstrated a statistically significant difference between the two treatment groups $(P=0.0210)$.
A similar comparison of central corneal staining after recovery from the $\mathrm{CAE}$ exposure (visits 2 and 5) also showed a significant reduction in fluorescein staining (Figure 3 ). The mean difference in staining for the $0.1 \% \mathrm{~T} \beta 4$-treated subjects was decreased by 0.37 , while the mean for subjects in the placebo group increased by 0.16 ; this difference was statistically significant $(P=0.0075)$. Peripheral (the mean superior and inferior cornea together) corneal staining also was significantly less in $0.1 \% \mathrm{~T} \beta 4$-treated eyes after 28 days of treatment in two separate analyses. The change from visit 1 post-CAE to visit 4 post-CAE was -0.19 in the $0.1 \%$ T $\beta 4$-treated eyes and +0.13 in the placebo-treated eyes $(P=0.0379)$. A summary of the corneal staining data is provided in Table 3.

As expected, ocular discomfort scores increased over the course of the CAE exposures for all treatment groups. Following the 28 -day treatment with $0.1 \% \mathrm{~T} \beta 4$, however, there was a distinction between the active and placebo treatment groups. From the beginning to the end of the CAE, discomfort scores rose from 1.3 to 3.5 in the placebo group but only rose from 1.7 to 3.3 in the $0.1 \%$ T $\beta 4$-treated group; comparison of the changes show a significantly lower increase for $0.1 \%$ T $\beta 4$-treated subjects ( $P=0.0224$, Figure 4$)$. 


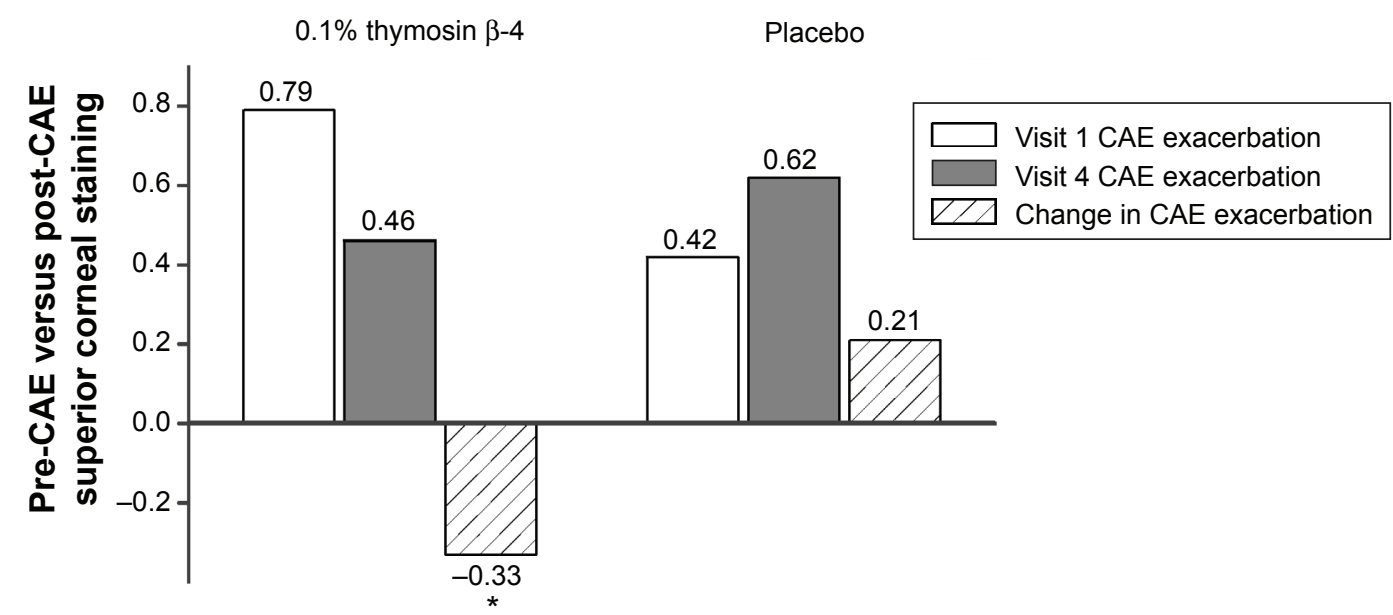

Figure 2 Reduction of superior corneal fluorescein staining by T $\beta 4$ between visit I and visit 4 . Mean values for staining exacerbation in the CAE were reduced in T $\beta 4$-treated subjects when compared with placebo-treated subjects.

Note: $* P=0.021$.

Abbreviation: CAE, controlled adverse environment.

No safety issues arose in this adverse environment challenge study with the use of $0.1 \% \mathrm{~T} \beta 4$ or its vehicle over the duration of treatment. No clinically significant changes in visual acuity, slit-lamp, and funduscopic assessments or intraocular pressure and corneal sensitivity measurements were observed. There was also a very low rate of TEAEs; only two of these were suspected to be related to study treatment, which was placebo in both cases. Only one subject was withdrawn from the study due to an adverse event, ie, moderately severe vomiting, suspected to be unrelated to the study treatment.

The total number of subjects with at least one ocular TEAE was two (5.6\%) in the $0.1 \% \mathrm{~T} \beta 4$-treated group and five
(13.9\%) in the placebo group. Both of the $0.1 \% \mathrm{~T} \beta 4$ events were classified as mild (5.6\%), while three were mild (8.3\%) and two moderate (5.6\%) in the placebo group. When broken down, one incidence of mild eye pain ( $2.8 \%$ each) occurred in each of the $0.1 \% \mathrm{~T} \beta 4$ and placebo groups. One mild blurred vision (2.8\%) was reported in the $0.1 \% \mathrm{~T} \beta 4$ group, and one mild eye irritation in the placebo group (2.8\%). A mild decrease in visual acuity was reported once (2.8\%) in the placebo-treated group, and a mild hordeolum was reported once (2.8\%) in the T $\beta 4$-treated group. Instillation site pain was reported twice in the placebo-treated group (5.6\%), one of which was mild (2.8\%) and one was moderate (2.8\%).

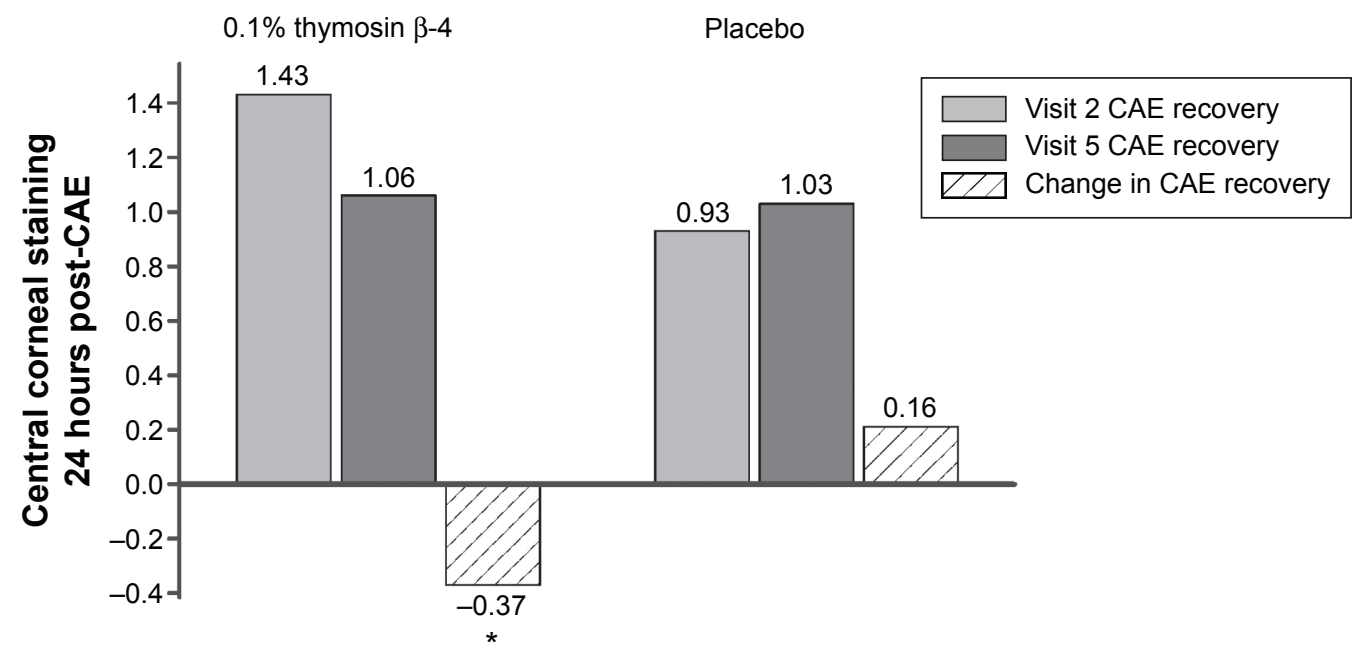

Figure 3 Reduction of central corneal fluorescein staining by T $\beta 4$ between visit 2 (baseline after CAE) and visit 5 (24-hour follow up after CAE). The data show a statistically significant improvement by T $\beta 4$ in healing and protection from exacerbation.

Note: $* P=0.0075$.

Abbreviation: CAE, controlled adverse environment. 
Table 3 Corneal staining by region

\begin{tabular}{|c|c|c|c|c|c|}
\hline Region & & Superior & Inferior & Peripheral & Central \\
\hline \multicolumn{6}{|l|}{ Treatment comparison } \\
\hline Mean Change (SD) post-CAE & Placebo & $+0.32(0.815)$ & $-0.06(0.886)$ & $+0.13(0.691)$ & $+0.65(0.942)$ \\
\hline Visit I to Visit 4 & $0.1 \% \mathrm{~T} \beta 4$ & $-0.14(0.648)$ & $-0.24(0.826)$ & $-0.19(0.575)$ & $+0.53(1.09)^{c}$ \\
\hline Mean Difference & & -0.47 & -0.18 & -0.33 & -0.12 \\
\hline$P$-value ${ }^{\mathrm{a}}$ & & 0.0109 & 0.3755 & 0.0379 & 0.6304 \\
\hline$P$-value ${ }^{\mathrm{b}}$ & & 0.0181 & 0.3478 & 0.0741 & 0.7322 \\
\hline Mean Change (SD) 24 hour post-CAE & Placebo & - & $-0.4(1.23)$ & - & $0.16(0.682)$ \\
\hline Visit 2 to Visit 5 & $0.1 \% \mathrm{~T} \beta 4$ & - & $-0.4(1.08)$ & - & $-0.36(0.87 I)$ \\
\hline Mean Difference & & & 0.00 & & -0.52 \\
\hline$P$-value ${ }^{\mathrm{a}}$ & & & 1.0000 & & 0.0075 \\
\hline$P$-value ${ }^{b}$ & & & 0.9186 & & 0.0066 \\
\hline
\end{tabular}

Notes: ${ }^{a}$-value calculated using a two-sample $t$-test assuming unequal variances comparing T $\beta 4$ to placebo. ${ }^{b} P$-value calculated using a Wilcoxon rank sum test comparing T $\beta 4$ to placebo. 'For central staining, comparison is Visit I pre-CAE to Visit 4 post-CAE. Bold values indicate. Significant differences between the placebo and $0.1 \%$ T $\beta 4$ are highlighted in bold.

Abbreviations: CAE, controlled adverse environment; SD, standard deviation.

A moderate instillation site reaction was also reported in the placebo-treated group once $(2.8 \%)$.

\section{Discussion}

In the present Phase II clinical trial, a 28-day course of $0.1 \%$ $\mathrm{T} \beta 4$ ophthalmic formulation elicited significant positive effects on ocular discomfort and on corneal staining in subjects with dry eye. Results suggest that $\mathrm{T} \beta 4$ has a protective effect, reducing the extent of corneal staining exhibited by subjects following exposure to a controlled adverse environment. In addition, significant differences between active and placebo groups at 24 hours post-CAE are consistent with an acceleration in healing for subjects treated with $\mathrm{T} \beta 4$.

A number of potential mechanisms may underlie these effects. ${ }^{3,4} \mathrm{~T} \beta 4$ has been shown to reduce production of

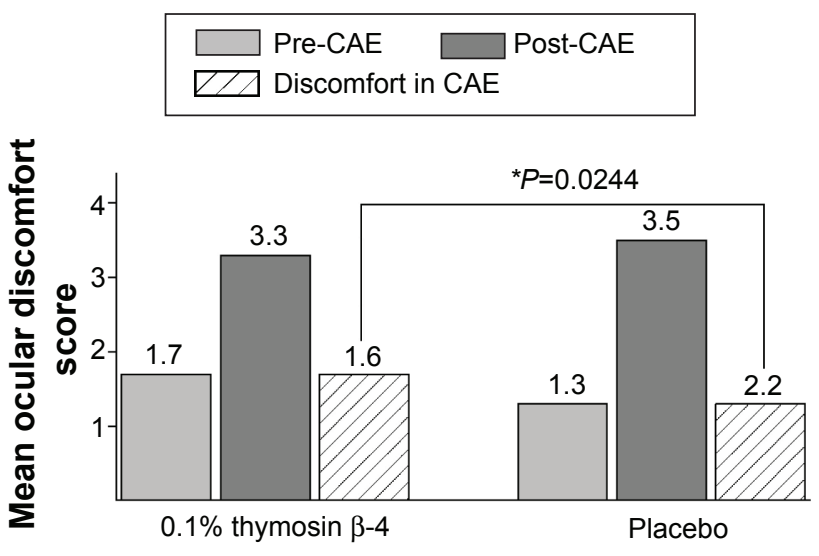

Figure 4 Reduction in CAE-associated ocular discomfort following 28 day treatment with $0.1 \% \mathrm{~T} \beta 4$. The change in mean discomfort scores in T $\beta 4$-treated subjects at visit 4 was statistically reduced when compared to mean placebo scores $(P=0.0244)$. Abbreviation: CAE, controlled adverse environment. inflammatory mediators and infiltration of inflammatory cells; these actions may reduce local concentrations of reactive oxygen species, thereby reducing local tissue damage and attenuating apoptotic activation., ${ }^{3,19,25}$ All of these effects would act to protect cells exposed to adverse stimuli, and would also potentially reduce activity in neural pathways associated with pain sensation. T $\beta 4$ has also been shown to regulate cellular actin dynamics and may enhance the cell motility, cell-matrix interactions, and cell-matrix remodeling that occurs during the course of wound healing. ${ }^{2,12}$

Another important finding from this study was the affirmation that therapeutic use of $\mathrm{T} \beta 4$ in a topical formulation is safe and well tolerated by users. No significant adverse events were reported, and no subjects withdrew from the study due to any adverse effect of the study formulation. Dry eye is a chronic disease, and future studies will need to address the long-term efficacy of $\mathrm{T} \beta 4$ as well as its long-term safety profile.

Two central features of dry eye that have made the development of new therapeutics challenging are the lack of correlation between signs and symptoms and the high degree of individual patient variability. ${ }^{1}$ This variability may contribute to the failure of comparisons between treatment groups to reach significance. Despite this, the findings of significant improvement for both a symptom (ocular discomfort) and a sign (corneal fluorescein staining), along with a lack of any drug-related adverse events, establish that $\mathrm{T} \beta 4$ has the potential to be a new, potent, and useful treatment for dry eye. Optimization of treatment regimens and study protocols going forward should allow future studies to confirm and extend these positive preliminary findings. 


\section{Disclosure}

The authors report no conflicts of interest in this work.

\section{References}

1. [No authors listed]. Report of the International Dry Eye Workshop (DEWS). Ocul Surf. 2007;5:65-204.

2. Crockford D, Turjman N, Allan C, Angel J. Thymosin beta4: structure, function, and biological properties supporting current and future clinical applications. Ann N Y Acad Sci. 2010;1194:179-189.

3. Goldstein AL, Hannappel E, Sosne G, Kleinman HK. Thymosin beta 4: a multifunctional regenerative peptide. Basic properties and clinical applications. Expert Opin Biol Ther. 2012;12:37-51.

4. Huff T, Müller CS, Otto AM, Netzker R, Hannappel E. Beta-thymosins, small acidic peptides with multiple functions. Int J Biochem Cell Biol. 2010;33:205-220.

5. Low TL, Goldstein AL. Chemical characterization of thymosin beta 4 . J Biol Chem. 1982;257:1000-1006.

6. Bock-Marquette I, Saxena A, White MD, et al. Thymosin $\beta 4$ activates integrin-linked kinase and promotes cardiac cell migration, survival and cardiac repair. Nature. 2004;432:466-472.

7. Malinda KM, Sidhu GS, Mani H, et al. Thymosin $\beta 4$ accelerates wound healing. J Invest Dermatol. 1999;113:364-368.

8. Sosne G, Siddiqi A, Kurpakus-Wheater M. Thymosin- $\beta 4$ inhibits corneal epithelial cell apoptosis after ethanol exposure in vitro. Invest Ophthalmol Vis Sci. 2004;45:1095-1100.

9. Morris D, Chopp M, Zhang L, et al. T $\beta 4$ Improves functional neurological outcome in a rat model of embolic stroke. Neuroscience. 2010;169: 674-682.

10. Sosne G, Hafeez S, Greenberry A 2nd, Kurpakus-Wheater M. Thymosin beta 4 promotes human conjunctival epithelial cell migration. Curr Eye Res. 2002;24:268-273.

11. Sosne G, Sliter EA, Barrett R, et al. Thymosin $\beta 4$ promotes corneal wound healing and decreases inflammation in vivo following alkali injury. Exp Eye Res. 2002;74:293-299.

12. Sosne G, Xu L, Prach L, et al. Thymosin $\beta 4$ stimulates laminin-5 production independent of TGF-beta. Exp Cell Res. 2004;293:175-183.

13. Zhang J, Zhang ZG, Morris D, et al. Neurological function recovery after T $\beta 4$ treatment in mice with experimental auto encephalomyelitis. Neuroscience. 2009;164:1887-1893.
14. Philp D, Badamchian M, Scheremeta B, et al. Thymosin beta 4 and a synthetic peptide containing its actin-binding domain promote dermal wound repair in $\mathrm{db} / \mathrm{db}$ diabetic mice and in aged mice. Wound Repair Regen. 2003;11:19-24.

15. Sosne G, Qiu P, Christopherson PL, Wheater MK. Thymosin $\beta 4$ suppression of corneal NF $\kappa \mathrm{B}$. A potential anti-inflammatory pathway. Exp Eye Res. 2007;84:663-669.

16. Hinkel R, El-Aouni C, Olson T, et al. Thymosin beta4 is an essential paracrine factor of embryonic endothelial progenitor cell-mediated cardioprotection. Circulation. 2008;117:2232-2240.

17. Smart N, Risebro CA, Melville AA, et al. Thymosin $\beta 4$ induces adult epicardial progenitor mobilization and neovascularization. Nature. 2007;445:177-182.

18. Sosne G, Chan CC, Thai K, et al. Thymosin beta 4 promotes corneal wound healing and modulates inflammatory mediators in vivo. Exp Eye Res. 2001;72:605-608.

19. Sosne G, Christopherson PL, Barrett RP, Friedman R. Thymosin-beta4 modulates corneal matrix metalloproteinase levels and polymorphonuclear cell infiltration after alkali injury. Invest Ophthalmol Vis Sci. 2005;46:2388-2395.

20. Allan CB, Cockcroft D, Crawford KS, Belen LJ. Effects of thymosin $\beta 4$ in a murine $\mathrm{CAE}^{\mathrm{TM}}$ model of experimental dry eye. Invest Ophthalmol Vis Sci. 2011;52:E3782.

21. Dunn SP, Heidemann DG, Chow CY, et al. Treatment of chronic nonhealing neurotrophic corneal epithelial defects with thymosin $\beta 4$. Ann NY Acad Sci. 2010;1194:199-206.

22. Sosne G, Dunn S, Crockford D, Kim C, Dixon E. Thymosin beta 4 eye drops significantly improve signs and symptoms of severe dry eye in a physician-sponsored phase-2 clinical trial. Invest Ophthalmol Vis Sci. 2013;54:E6033.

23. Ruff D, Crockford D, Girardi G, Zhang Y. A randomized, placebocontrolled, single and multiple dose study of intravenous thymosin beta4 in healthy volunteers. Ann N Y Acad Sci. 2010;1194:223-229.

24. Abelson R, Lane KJ, Rodriguez J, et al. A single-center study evaluating the effect of the controlled adverse environment $\left(\mathrm{CAE}^{\mathrm{TM}}\right)$ model on tear film stability. Clin Ophthalmol. 2012;6:1865-1872.

25. Wilson SE, Mohan RR, Mohan RR, et al. The corneal wound healing response; cytokine mediated interaction of the epithelium, stroma and inflammatory cells. Prog Retin Eye Res. 2001;20:625-637.
Clinical Ophthalmology

\section{Publish your work in this journal}

Clinical Ophthalmology is an international, peer-reviewed journal covering all subspecialties within ophthalmology. Key topics include: Optometry; Visual science; Pharmacology and drug therapy in eye diseases; Basic Sciences; Primary and Secondary eye care; Patient Safety and Quality of Care Improvements. This journal is indexed on Submit your manuscript here: http://www.dovepress.com/clinical-ophthalmology-journal
Dovepress

PubMed Central and CAS, and is the official journal of The Society of Clinical Ophthalmology (SCO). The manuscript management system is completely online and includes a very quick and fair peer-review system, which is all easy to use. Visit http://www.dovepress.com/ testimonials.php to read real quotes from published authors. 